Review began 09/23/2021 Review ended 09/28/2021 Published 09/30/2021

\section{() Copyright 2021}

Qayum et al. This is an open access article distributed under the terms of the Creative Commons Attribution License CC-BY 4.0., which permits unrestricted use, distribution, and reproduction in any medium, provided the original author and source are credited.

\title{
Single- Versus Double-Layer Uterine Closure After Cesarean Section Delivery: A Systematic Review and Meta-Analysis
}

\author{
Kaif Qayum ${ }^{1}$, Irfan $\operatorname{Kar}^{1}$, Junaid Sofi ${ }^{2}$, Hari Panneerselvam ${ }^{3}$ \\ 1. General Surgery, Wye Valley NHS Foundation Trust, Hereford, GBR 2. General Surgery, Sher-I-Kashmir Institute of \\ Medical Sciences, Srinagar, IND 3. Medicine, Wye Valley NHS Foundation Trust, Hereford, GBR
}

Corresponding author: Kaif Qayum, kaifqayum7@gmail.com

\begin{abstract}
Cesarean section (CS) delivery is a common procedure, and its incidence is increasing globally. To compare single-layer (SL) with double-layer (DL) uterine closure techniques after cesarean section in terms of ultrasonographic findings and rate of CS complications. PubMed, Scopus, Web of Science, and Cochrane Library were searched for relevant randomized clinical trials (RCTs). Retrieved articles were screened, and relevant studies were included in a meta-analysis. Continuous data were pooled as mean difference (MD) with $95 \%$ confidence interval (CI), and dichotomous data were pooled as relative risk (RR) and 95\% CI. Analysis was conducted using RevMan software (Version 5.4). Eighteen RCTs were included in our study. Pooled results favored DL uterine closure in terms of residual myometrial thickness ( $\mathrm{MD}=-1.15 ; 95 \% \mathrm{CI}$ $1.69,-0.60 ; \mathrm{P}<0.0001)$ and dysmenorrhea $(\mathrm{RR}=1.36 ; 95 \% \mathrm{CI} 1.02,1.81 ; \mathrm{P}=0.04)$, while SL closure had shorter operation time than DL closure $(\mathrm{MD}=-2.25 ; 95 \% \mathrm{CI}-3.29,-1.21 ; \mathrm{P}<0.00001)$. Both techniques had similar results in terms of uterine dehiscence or rupture ( $\mathrm{RR}=1.88 ; 95 \% \mathrm{CI} 0.63,5.62 ; \mathrm{P}=0.26)$, healing ratio $(\mathrm{MD}=-5.00 ; 95 \% \mathrm{CI}-12.40,2.39 ; \mathrm{P}=0.18)$, maternal infectious morbidity $(\mathrm{RR}=0.94 ; 95 \% \mathrm{CI} 0.66,1.34$; $\mathrm{P}=0.72)$, hospital stay $(\mathrm{MD}=-0.12 ; 95 \% \mathrm{CI}-0.30,0.06 ; \mathrm{P}=0.18)$, and readmission rate $(\mathrm{RR}=0.95 ; 95 \% \mathrm{CI}$ $0.64,1.40 ; \mathrm{P}=0.78$ ). Double-layer uterine closure shows more residual myometrial thickness and lower incidence of dysmenorrhea than single-layer uterine closure of cesarean section scar. But single-layer closure has the advantage of the shorter operation time. Both methods have comparable blood loss amount, healing ratio, hospital stay duration, maternal infection risk, readmission rate, and uterine dehiscence or rupture risk.
\end{abstract}

Categories: Obstetrics/Gynecology

Keywords: double-layer, single-layer, uterine closure, residual myometrium thickness, cesarean section

\section{Introduction And Background}

Cesarean section (CS) incidence is increasing globally through recent years, reaching $25 \%$ of total deliveries in some countries [1]. This rise in the incidence of CS increases the events of CS-related complications [2]. CS complications include infection, hemorrhage and thromboembolism as short-term complications in addition to the long-term complications and symptoms including dysmenorrhea, dysuria, abnormal uterine bleeding, and infertility [2,3]. Some CS complications - such as placenta accreta, uterine rupture or dehiscence, and CS scar pregnancy - may be manifested during a subsequent pregnancy due to a defective uterine scar [3]. The prevalence of uterine scar defect in women with previous CS is unexpectedly high, ranging from $56 \%$ to $84 \%$ when examined by transvaginal ultrasonography with contrast [4].

In pregnant women with a previous cesarean delivery, the risk of uterine rupture during a subsequent trial of labor has to be assessed. Its assessment is done using ultrasonographic measurement of the lower uterine segment and the residual myometrial thickness (RMT) [5]. Defective RMT was linked to a higher risk of adverse outcomes, including postmenstrual spotting, uterine dehiscence or rupture, placental adherence, failure of labor trial, and more complications of CS scar pregnancy [6]. It has been hypothesized that uterine incision closure technique may be associated with the development of the uterine niche and subsequent CSrelated adverse outcomes [4].

The surgical method of uterine closure after CS is suggested to affect the RMT, uterine scar defect, and the healing of the uterine scar. However, clear evidence of the best method for uterine closure is not established [7], and no evidence-based guideline for the closure technique is present [8]. Previous studies reported that double-layer (DL) closure has thicker residual myometrium and a lower incidence of large defects than single-layer (SL) closure. However, a clear conclusion about other clinical outcomes is still lacking [9].

This systematic review and meta-analysis aim to compare the ultrasonographic findings and complication rate of single-layer (SL) versus double-layer (DL) uterine closure techniques after CS procedure.

\section{Review}




\section{Methods}

This systematic review and meta-analysis followed the steps described in the "Preferred Reporting Items for Systematic reviews and Meta-Analyses (PRISMA)" and in the "Cochrane handbook for systematic reviews of interventions" [10,11].

Data Collection and Search Strategy

We searched PubMed, Scopus, Web of Science, and Cochrane library databases for published randomized control trials (RCTs) from inception till June 2021. We used the following keywords: "surgical technique," "endometrium," "suture technique," "single-layer," "double-layer," "cesarean section," and "postcesarean." We applied no restrictions regarding age, publication date, the indication of cesarean section, or the number of previous deliveries.

Inclusion and Exclusion Criteria

We included RCTs that compared SL versus DL uterine closure techniques after cesarean section delivery and reported any of the ultrasonographic outcomes or adverse events. We excluded observational studies, reviews, non-randomized trials, cross-sectional studies, editorials, abstracts, thesis, letters, books, and chapters.

Screening and Study Selection

Retrieved records were imported to Endnote software, and duplicates were removed. The remaining records underwent title and abstract screening then full-text screening according to our eligibility criteria. Three reviewers performed the screening process independently, and any disagreement was solved by discussion. Eligible articles were included in the meta-analysis.

\section{Data Extraction}

All study authors shared in the data extraction. We extracted data related to the following domains: (1) summary of the included studies, including the study name, national clinical trial (NCT) number registration number, country where the study was carried out, sample size, period of follow-up, and study outcomes, (2) baseline characters of the included studies' population, including study arms, age of the participant, gestational age at delivery, body mass index, birth weight, nulliparity, preterm delivery, multiple pregnancy, elective cesarean delivery, and prior cesarean deliveries, (3) outcomes, including residual myometrial thickness, dysmenorrhea, uterine dehiscence or rupture, healing ratio, blood loss, operative time, maternal infectious morbidity, hospital stay, and readmission rate, and (4) quality assessment domains.

Quality Assessment

According to the Cochrane Collaboration tool for risk of bias assessment in randomized studies, we evaluated the quality of the included studies [12]. The tool included the judgment of the selection, performance, detection, attrition, reporting, and other bias domains. Each domain was judged as low, high, or unclear risk of bias. At least two independent reviewers judged each domain and conflicts were solved by discussion.

\section{Statistical Analysis}

Data analysis was conducted using review manager (RevMan) software version 5.4. Data of continuous outcomes were reported as mean difference (MD) and 95\% confidence interval (CI) using the InverseVariance method, and dichotomous data were reported as relative risk (RR) and 95\% CI using the MantelHaenszel method. We assessed heterogeneity using chi-square and I-square tests, and heterogeneity was considered significant at chi-square P-value $<0.1$ and $\mathrm{I} 2>50 \%$. We used the random-effects model for analysis. Whenever pooled data are heterogeneous, we tried to solve the heterogeneity by sensitivity analysis using the leave-one-out test and subgroup analysis. We performed a subgroup analysis, when applicable, according to the used suturing technique, whether locked or unlocked sutures, and whether the decidua is included or excluded from suturing.

\section{Results}

Literature Search and Study Selection

Searching electronic databases yielded a total of 3926 articles. After removing duplicates, we had 3018 unique articles that underwent title and abstract screening. Of these articles, 2907 were excluded, and 111 full-texts were retrieved and screened according to our eligibility criteria. Finally, 18 studies were considered eligible for inclusion in the meta-analysis [3,8,13-28]. Figure 1 summarizes the flow of the study selection process and data collection. 


\section{Cureus}
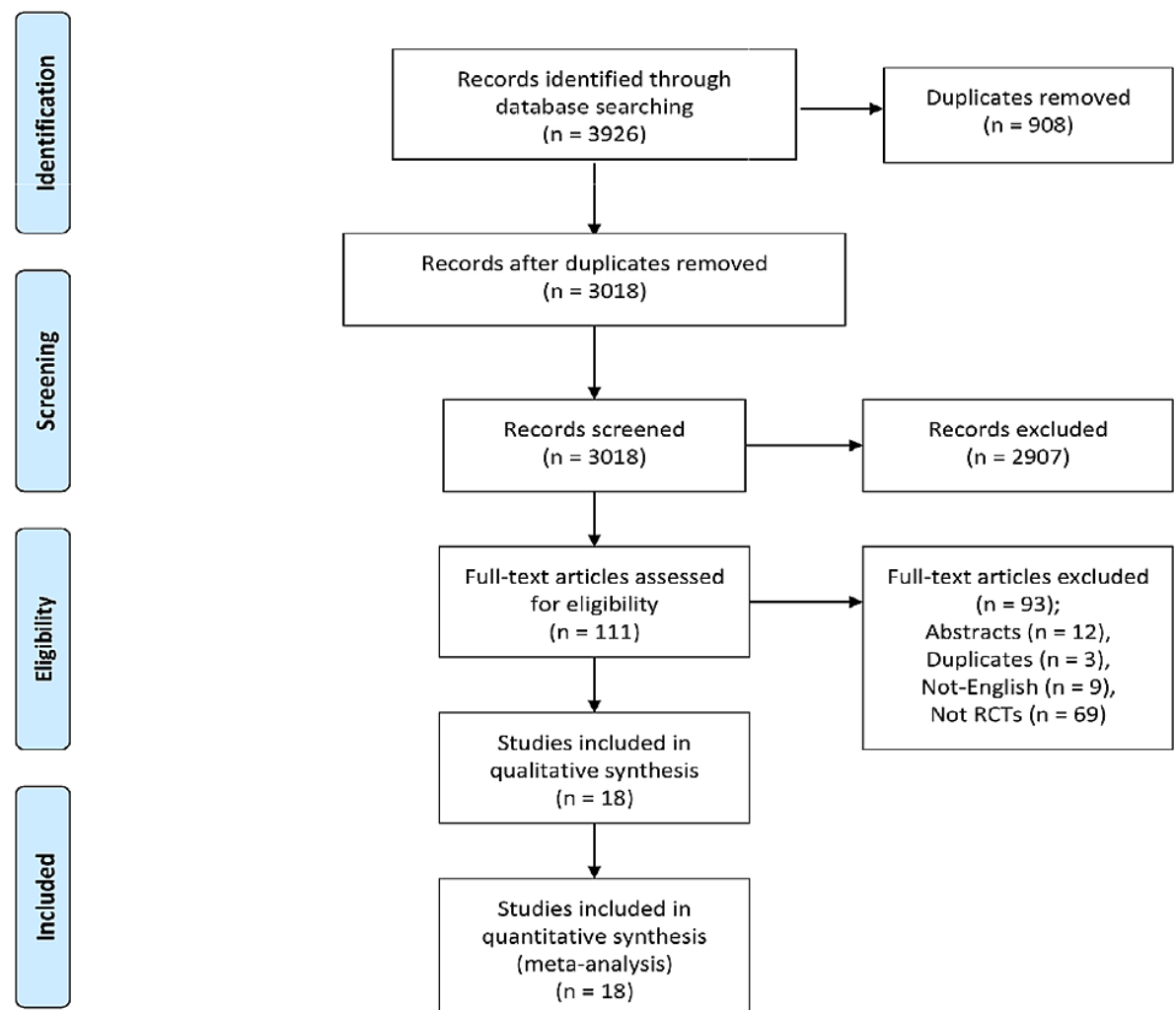
qualitative synthesis

$$
(n=18)
$$

Studies included in

quantitative synthesis (meta-analysis)

$$
(n=18)
$$

FIGURE 1: PRISMA flow chart summarizing the process of data collection and study selection.

PRISMA: Preferred Reporting Items for Systematic reviews and Meta-Analyses.

Characteristics of the Included Studies

Included studies were performed in various countries. The sample size varied considerably across studies, ranging from 30 to 7411 . The follow-up period varied from six weeks in some studies to 6-24 months in other studies. The mean age of included patient groups ranged from 24 to 32 years, while mean gestational age ranged from 37.8 to 40 weeks. Table 1 and Table 2 show the summary of included studies and the baseline characters of included patients, respectively. 


\section{Cureus}

\begin{tabular}{|c|c|c|c|c|c|}
\hline ID & Country & NCT & $\begin{array}{l}\text { Sample } \\
\text { size }\end{array}$ & $\begin{array}{l}\text { Follow- } \\
\text { up }\end{array}$ & Outcomes \\
\hline $\begin{array}{l}\text { Bamberg } 2016 \\
{[14]}\end{array}$ & Germany & $\begin{array}{l}\text { NCT } \\
02338388\end{array}$ & 306 & $6-24 \mathrm{M}$ & $\begin{array}{l}\text { Residual myometrium thickness, blood loss, operative time, } \\
\text { maternal infectious morbidity }\end{array}$ \\
\hline $\begin{array}{l}\text { Bennich } 2016 \\
{[15]}\end{array}$ & Denmark & NCT02144805 & 76 & $5 \mathrm{M}$ & $\begin{array}{l}\text { Residual myometrium thickness, dysmenorrhea, healing ratio, blood } \\
\text { loss, operative time }\end{array}$ \\
\hline $\begin{array}{l}\text { CAESAR } 2010 \\
{[13]}\end{array}$ & Multicenter & $\begin{array}{l}\text { ISRCTN } \\
11849611\end{array}$ & 2979 & $6 \mathrm{~W}$ & $\begin{array}{l}\text { Operative time, maternal infectious morbidity, hospital stay, } \\
\text { readmission rate }\end{array}$ \\
\hline $\begin{array}{l}\text { Chapman } 1997 \\
\text { [16] }\end{array}$ & $\begin{array}{l}\text { United } \\
\text { States }\end{array}$ & - & 145 & $4 \mathrm{Y}$ & Uterine rupture, hospital stay \\
\hline $\begin{array}{l}\text { CORONIS } \\
2016[3]\end{array}$ & International & $\begin{array}{l}\text { OXTREC; } \\
013-06 a\end{array}$ & 7411 & $3 Y$ & Dysmenorrhea, uterine rupture \\
\hline $\begin{array}{l}\text { El-Gharib } 2013 \\
\text { [18] }\end{array}$ & Egypt & - & 150 & $6 \mathrm{~W}$ & $\begin{array}{l}\text { Residual myometrium thickness, operative time, maternal infectious } \\
\text { morbidity, hospital stay }\end{array}$ \\
\hline $\begin{array}{l}\text { Hamar } 2007 \\
{[19]}\end{array}$ & $\begin{array}{l}\text { United } \\
\text { States }\end{array}$ & NCT00224250 & 30 & $6 \mathrm{~W}$ & Residual myometrium thickness, blood loss, operative time \\
\hline $\begin{array}{l}\text { Hanacek } 2019 \\
\text { [20] }\end{array}$ & $\begin{array}{l}\text { Czech } \\
\text { Republic }\end{array}$ & - & 540 & $12 \mathrm{M}$ & Residual myometrium thickness, maternal infectious morbidity \\
\hline $\begin{array}{l}\text { Hauth } 1992 \\
\text { [21] }\end{array}$ & $\begin{array}{l}\text { United } \\
\text { States }\end{array}$ & - & 906 & - & Maternal infectious morbidity \\
\hline $\begin{array}{l}\text { Kalem } 2019 \\
{[22]}\end{array}$ & Turkey & - & 138 & - & Residual myometrium thickness, dysmenorrhea, operative time \\
\hline $\begin{array}{l}\text { Khamees } 2018 \\
{[17]}\end{array}$ & Egypt & - & 80 & - & Residual myometrium thickness, blood loss, operative time \\
\hline $\begin{array}{l}\text { Roberge } 2016 \\
\text { [24] }\end{array}$ & Canada & NCT01860859 & 54 & $6-12 \mathrm{M}$ & $\begin{array}{l}\text { Residual myometrium thickness, healing ratio, blood loss, operative } \\
\text { time, maternal infectious morbidity }\end{array}$ \\
\hline $\begin{array}{l}\text { Sevket } 2014 \\
{[25]}\end{array}$ & Turkey & - & 36 & $6 \mathrm{M}$ & $\begin{array}{l}\text { Residual myometrium thickness, healing ratio, blood loss, operative } \\
\text { time }\end{array}$ \\
\hline $\begin{array}{l}\text { Shrestha } 2015 \\
\text { [26] }\end{array}$ & Nepal & - & 50 & $6 \mathrm{~W}$ & Residual myometrium thickness \\
\hline Sood 2005 [23] & India & - & 208 & $6 \mathrm{~W}$ & $\begin{array}{l}\text { Blood loss, operative time, maternal infectious morbidity, hospital } \\
\text { stay }\end{array}$ \\
\hline $\begin{array}{l}\text { Stegwee } 2020 \\
{[8]}\end{array}$ & Netherlands & 2015.462 & 2852 & $9 \mathrm{M}$ & $\begin{array}{l}\text { Residual myometrium thickness, healing ratio, blood loss, operative } \\
\text { time, hospital stay, readmission rate }\end{array}$ \\
\hline $\begin{array}{l}\text { Yasmin } 2011 \\
{[27]}\end{array}$ & Pakistan & - & 60 & $6 \mathrm{~W}$ & $\begin{array}{l}\text { Residual myometrium thickness, uterine rupture, blood loss, } \\
\text { operative time }\end{array}$ \\
\hline $\begin{array}{l}\text { Yilmazbaran } \\
2020[28]\end{array}$ & Turkey & NCT03629028 & 282 & 6-9 M & metrium thickness, dysmenorrhea, operative time \\
\hline
\end{tabular}

\section{TABLE 1: Summary of the included studies.}

W, weeks; M, months; Y, years; NCT, National Clinical Trial; ISRCTN, International Standard Randomized Controlled Trial; OXTREC, Oxford Tropical Research Ethics Committee.

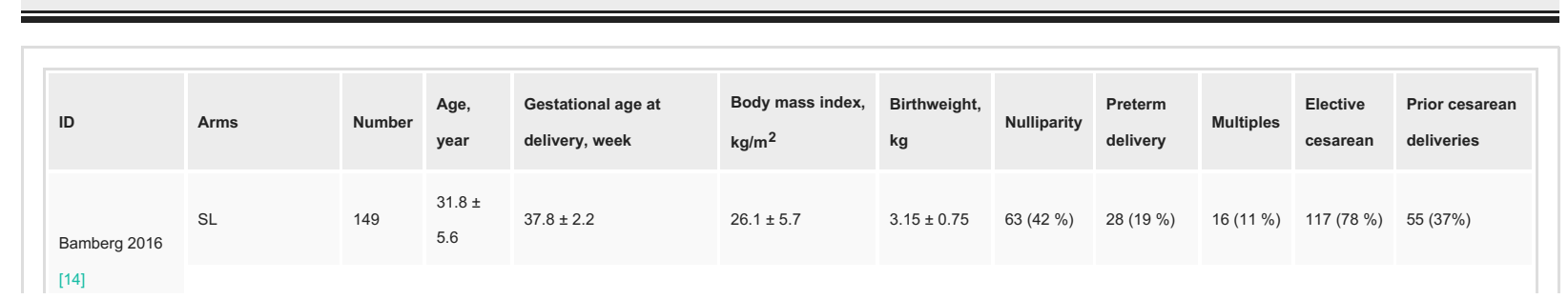




\section{Cureus}

\begin{tabular}{|c|c|c|c|c|c|c|c|c|c|c|c|}
\hline & DL & 129 & $\begin{array}{l}30.3 \pm \\
6.5\end{array}$ & $37.3 \pm 2.3$ & $25.6 \pm 6.2$ & $3.09 \pm 0.67$ & $48(37 \%)$ & $25(19 \%)$ & $13(10 \%)$ & $103(79 \%)$ & $57(44 \%)$ \\
\hline \multirow{2}{*}{$\begin{array}{l}\text { Bennich } 2016 \\
{[15]}\end{array}$} & SL & 35 & $\begin{array}{l}30.3 \pm \\
4.5\end{array}$ & $38.7 \pm 0.6$ & $24.6 \pm 4.8$ & - & $35(47.9)$ & - & - & $35(47.9)$ & - \\
\hline & $\mathrm{DL}$ & 38 & $\begin{array}{l}30.5 \pm \\
5.5\end{array}$ & $38.9 \pm 0.7$ & $24.1 \pm 3.5$ & - & $38(52.1)$ & - & - & $38(52.1)$ & - \\
\hline \multirow{2}{*}{$\begin{array}{l}\text { CAESAR } 2010 \\
{[13]}\end{array}$} & SL & 1483 & $\begin{array}{l}30.6 \pm \\
5.9\end{array}$ & $39.0 \pm 2.0$ & - & - & $989(67)$ & - & 79 & - & - \\
\hline & $\mathrm{DL}$ & 1496 & $\begin{array}{l}30.6 \pm \\
5.9\end{array}$ & $39.1 \pm 1.9$ & - & - & $480(32)$ & - & 76 & - & - \\
\hline \multirow{2}{*}{$\begin{array}{l}\text { Chapman } 1997 \\
\text { [16] }\end{array}$} & SL & 70 & - & $37 \pm 5.2$ & - & - & - & 14 & - & - & - \\
\hline & $\mathrm{DL}$ & 75 & - & $40 \pm 3.7$ & - & - & - & 25 & - & - & - \\
\hline \multirow{2}{*}{$\begin{array}{l}\text { CORONIS } 2016 \\
\text { [3] }\end{array}$} & $\mathrm{SL}$ & 4705 & - & - & - & - & - & - & - & - & - \\
\hline & $\mathrm{DL}$ & 4711 & - & - & - & - & - & - & - & - & - \\
\hline \multirow{2}{*}{$\begin{array}{l}\text { El-Gharib } 2013 \\
\text { [18] }\end{array}$} & SL & 75 & $\begin{array}{l}28.84 \pm \\
3.4\end{array}$ & $39.11 \pm 0.7$ & - & $2.86 \pm 0.6$ & $75(100 \%)$ & - & - & - & $0(0 \%)$ \\
\hline & DL & 75 & $\begin{array}{l}28.36 \pm \\
3.2\end{array}$ & $39.16 \pm 0.7$ & - & $2.87 \pm 0.6$ & $75(100 \%)$ & - & - & - & $0(0 \%)$ \\
\hline \multirow{2}{*}{$\begin{array}{l}\text { Hamar } 2007 \\
\text { [19] }\end{array}$} & SL & 15 & $30 \pm 7$ & $39.3 \pm 0.5$ & - & $3.35 \pm 0.75$ & $11(73 \%)$ & - & - & - & - \\
\hline & $\mathrm{DL}$ & 15 & $25 \pm 7$ & $38.6 \pm 0.9$ & - & $3.44 \pm 0.43$ & $8(53 \%)$ & - & - & - & - \\
\hline \multirow{2}{*}{$\begin{array}{l}\text { Hanacek } 2019 \\
\text { [20] }\end{array}$} & SL & 149 & $\begin{array}{l}31(29- \\
34)\end{array}$ & $40(39-41)$ & $22.4(20.4-25.3)$ & - & - & - & & - & - \\
\hline & DL & 175 & $\begin{array}{l}32(29- \\
34)\end{array}$ & $40(40-41)$ & $22.3(20.1-24.2)$ & - & - & - & & - & - \\
\hline \multirow{2}{*}{ Hauth 1992 [21] } & SL & 457 & 24.2 & 38 & - & - & $220(48 \%)$ & - & $16(6 \%)$ & - & $126(28 \%)$ \\
\hline & DL & 449 & 24.6 & 37.8 & - & - & $239(53 \%)$ & - & $20(4 \%)$ & - & $99(22 \%)$ \\
\hline \multirow{2}{*}{ Kalem 2019 [22] } & SL & 68 & $\begin{array}{l}29.25 \pm \\
6.27\end{array}$ & $38.5 \pm 2.7$ & $26.04 \pm 2.37$ & $3.23 \pm 0.51$ & - & - & - & - & - \\
\hline & $\mathrm{DL}$ & 70 & $\begin{array}{l}28.94 \pm \\
5.17\end{array}$ & $39.4 \pm 3.6$ & $25.90 \pm 2.28$ & $3.26 \pm 0.49$ & - & - & - & - & - \\
\hline \multirow{2}{*}{$\begin{array}{l}\text { Khamees } 2018 \\
{[17]}\end{array}$} & SL & 40 & - & - & - & - & $40(100 \%)$ & - & - & - & $0(0 \%)$ \\
\hline & DL & 40 & - & - & - & - & $40(100 \%)$ & - & - & - & $0(0 \%)$ \\
\hline \multirow{3}{*}{$\begin{array}{l}\text { Roberge } 2016 \\
{[24]}\end{array}$} & SL & 27 & $\begin{array}{l}30.8 \pm \\
4.0\end{array}$ & $39.2 \pm 0.6$ & $25.1 \pm 4.7$ & $3.35 \pm 0.379$ & 22 & - & - & - & - \\
\hline & $\begin{array}{l}\text { DL with locked } \\
\text { sutures }\end{array}$ & 27 & $\begin{array}{l}31.1 \pm \\
6.4\end{array}$ & $39.1 \pm 0.5$ & $23.5 \pm 3.9$ & $3.41 \pm 0.44$ & 20 & - & - & - & - \\
\hline & $\begin{array}{l}\text { DL with unlocked } \\
\text { sutures }\end{array}$ & 27 & $31 \pm 3.7$ & $38.9 \pm 0.6$ & $25.1 \pm 5.3$ & $3.24 \pm 0.47$ & 20 & - & - & - & - \\
\hline \multirow{2}{*}{$\begin{array}{l}\text { Sevket } 2014 \\
{[25]}\end{array}$} & SL & 15 & $\begin{array}{l}29.7 \pm \\
6.5\end{array}$ & $38.6 \pm 0.8$ & - & $3.44 \pm 0.43$ & & - & - & - & - \\
\hline & $\mathrm{DL}$ & 16 & $\begin{array}{l}29.4 \pm \\
7.3\end{array}$ & $39 \pm 1.2$ & - & $3.39 \pm 0.38$ & & - & - & - & - \\
\hline \multirow{3}{*}{$\begin{array}{l}\text { Shrestha } 2015 \\
{[26]}\end{array}$} & SL & 25 & $\begin{array}{l}26.04 \pm \\
5.06\end{array}$ & $38.36 \pm 2.21$ & - & - & $21(84 \%)$ & - & - & $16(64 \%)$ & - \\
\hline & $\mathrm{DL}$ & 25 & $\begin{array}{l}23.92 \pm \\
4.32\end{array}$ & $38.92 \pm 1.35$ & - & - & 17 (68\%) & - & - & $8(32 \%)$ & - \\
\hline & SL & 102 & $26.5 \pm$ & $38.2 \pm 1.5$ & - & - & - & - & - & $66(64.7 \%)$ & 34 (33.4\%) \\
\hline
\end{tabular}




\section{Cureus}

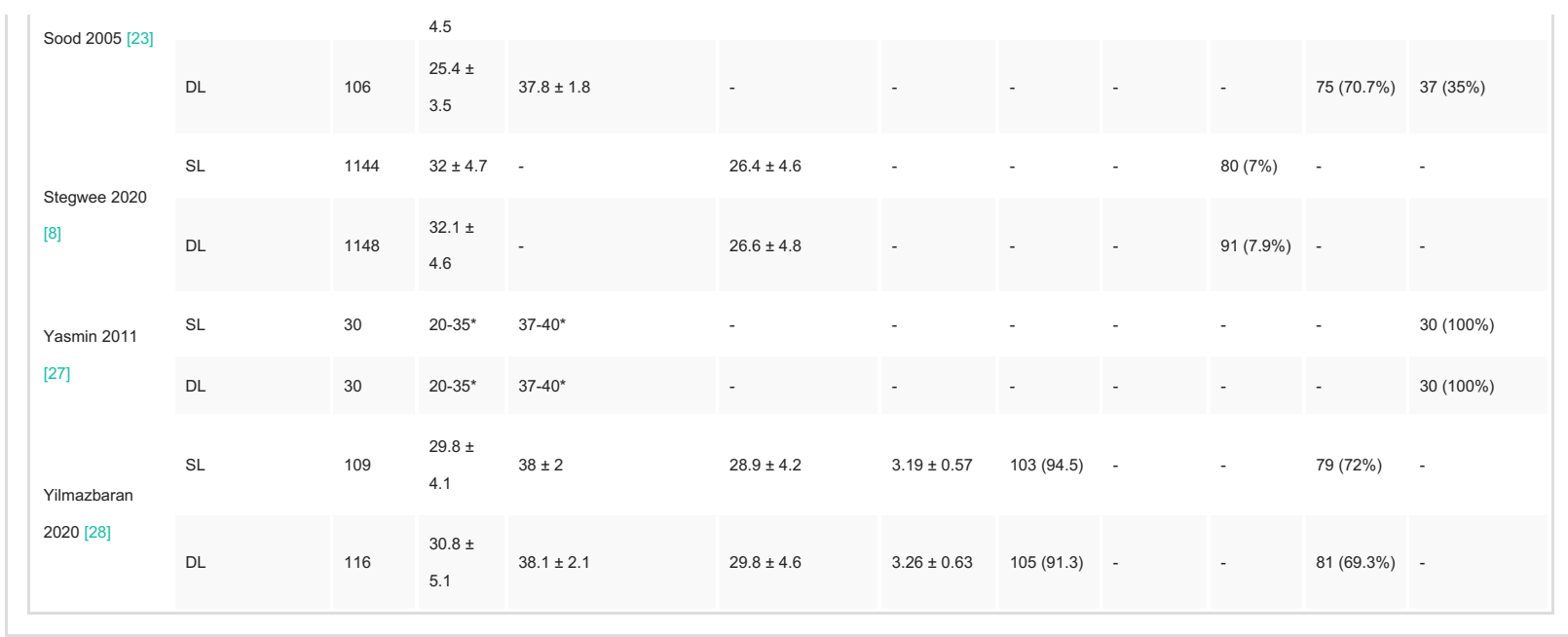

TABLE 2: Baseline characteristics of the included studies' population.

Data are presented as mean \pm standard deviation, median (interquartile range), or number (percentage).

${ }^{*}$ Range.

$\mathrm{SL}$, single-layer uterine closure; DL, double-layer uterine closure.

Quality Assessment

Most included studies had a low risk of selection bias regarding both selection bias domains: random sequence generation and allocation concealment. However, the remaining studies were of unclear risk of selection bias because the reported data are insufficient to judge. Most studies had an unclear risk of performance bias because they reported scarce details to judge the blinding process of participants and personnel. In contrast, detection bias was at low risk in most studies due to proper blinding of the outcome assessor. Attrition bias was at low risk in most studies because the lost data are insufficient to produce bias results. Reporting bias was judged low risk in most studies because the outcomes of interest were reported as expected. The "other bias" domain was judged low risk in most studies and unclear in some studies. The risk of bias graph shows the overall judgment of each risk of bias domain (Figure 2) and the risk of bias summary summarizes the judgment of each domain in each study (Figure 3).

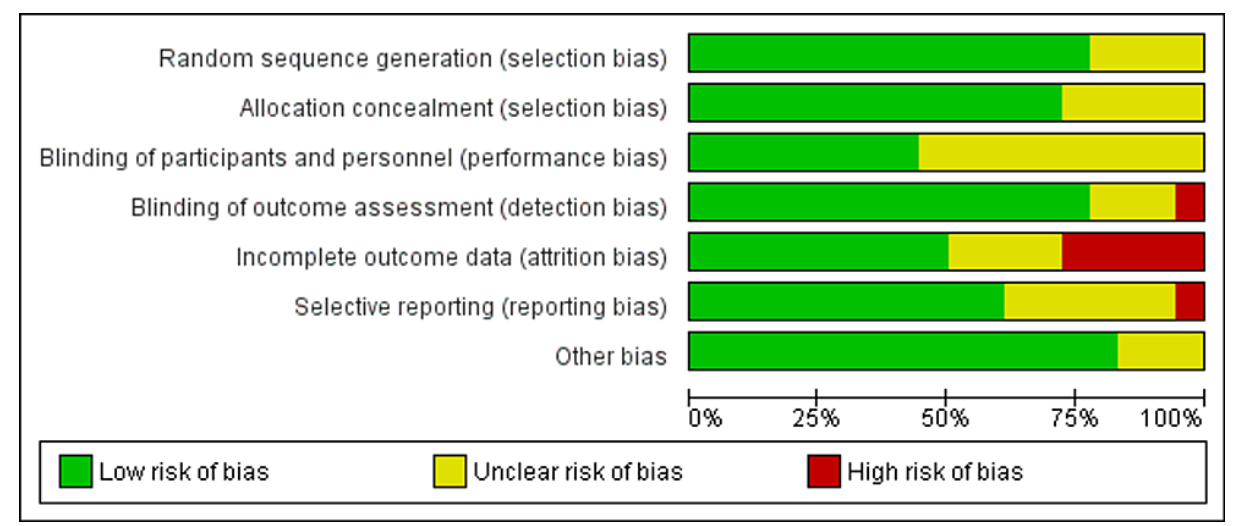

FIGURE 2: Risk of bias graph. 


\section{Cureus}

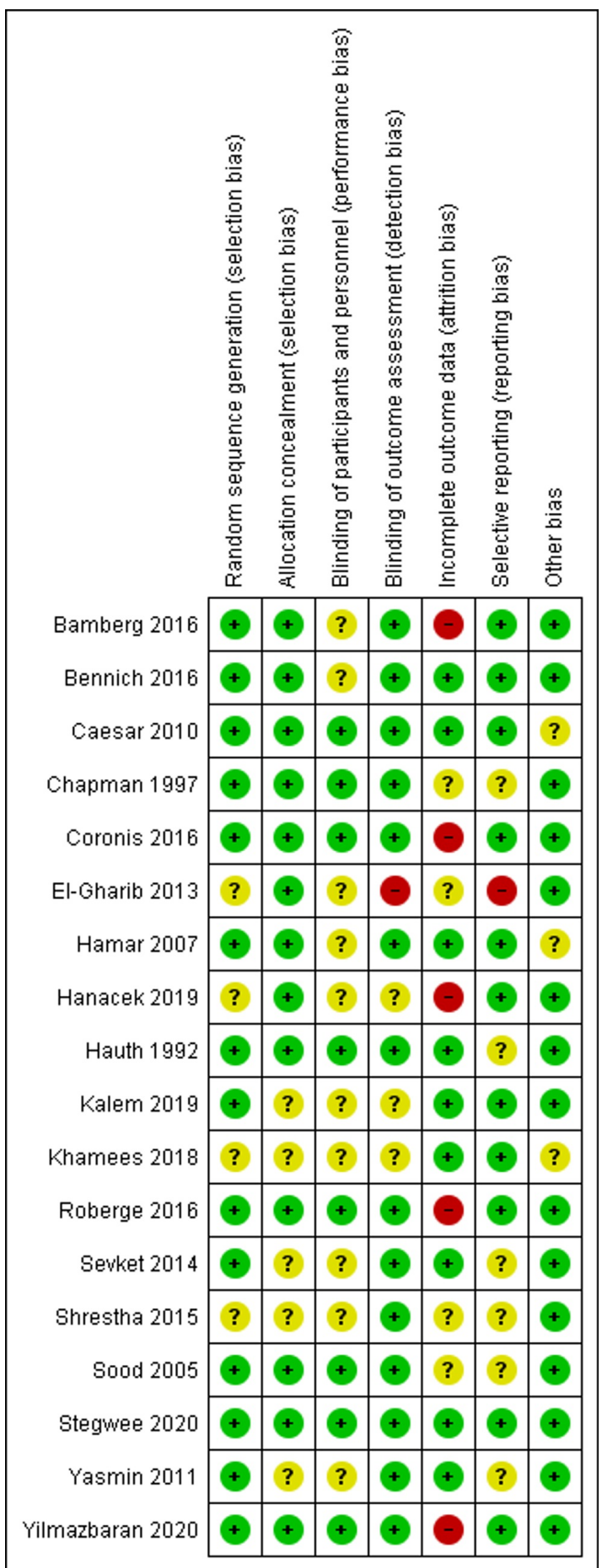

FIGURE 3: Risk of bias summary.

Outcomes

Residual myometrial thickness (Figure 4)

The residual myometrial thickness was significantly lower with the SL compared with the DL uterine closure technique $(\mathrm{MD}=-1.15 ; 95 \% \mathrm{CI}-1.69,-0.60 ; \mathrm{P}<0.0001)$. Pooled data are heterogeneous $(\mathrm{P}<0.00001 ; \mathrm{I} 2=$ $88 \%)$. In the subgroup of locked sutures with inclusion of the decidua, SL uterine closure showed lower RMT $(\mathrm{MD}=-1.10 ; 95 \% \mathrm{CI}-1.81,-0.38 ; \mathrm{P}=0.003)$ and the results were heterogeneous, but heterogeneity was solved after excluding Shrestha 2015 and the results remained significant. Pooled results were also lower with SL than DL uterine closure in the subgroup of locked sutures with no data about decidual layer inclusion ( $\mathrm{MD}=-2.51 ; 95 \% \mathrm{CI}-3.28,-1.75 ; \mathrm{P}<0.00001)$, and the results were homogeneous $(\mathrm{P}=0.36 ; \mathrm{I} 2=$ $0 \%)$. Also, in the subgroup of unlocked sutures with inclusion of the decidua, SL showed lower RMT than DL 


\section{Cureus}

uterine closure $(\mathrm{MD}=-0.64 ; 95 \% \mathrm{CI}-1.14,-0.13 ; \mathrm{P}=0.01)$ and the results were homogeneous $(\mathrm{P}=0.31 ; \mathrm{I} 2=$ $14 \%)$. Also, the subgroup analysis comparing SL closure using locked sutures versus DL closure using unlocked sutures showed no significant difference between both groups $(\mathrm{MD}=-2.24 ; 95 \% \mathrm{CI}-4.52,0.04 ; \mathrm{P}=$ $0.05)$ and the results were heterogeneous $(\mathrm{P}<0.00001 ; \mathrm{I}=96 \%)$.

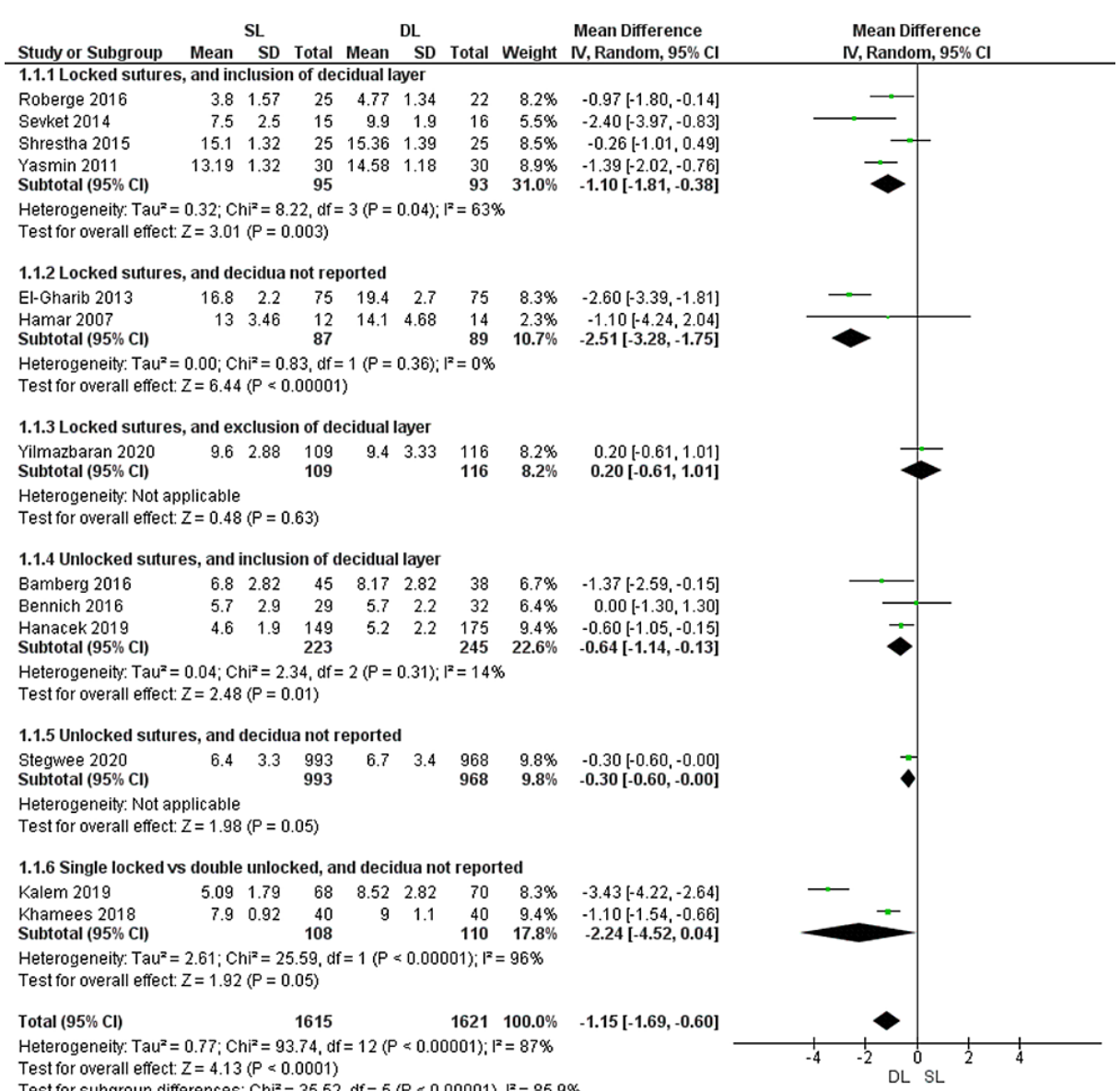

FIGURE 4: Forest plot comparing single- versus double-layer uterine closure in terms of residual myometrial thickness.

$\mathrm{SL}$, single-layer uterine closure; DL, double-layer uterine closure.

Dysmenorrhea (Figure 5)

Pooled data showed higher risk of dysmenorrhea with SL than DL uterine closure $(\mathrm{RR}=1.36 ; 95 \%$ CI 1.02 , $1.81 ; \mathrm{P}=0.04)$. Pooled data were homogeneous $(\mathrm{P}=0.33 ; \mathrm{I} 2=12 \%)$. 


\section{Cureus}

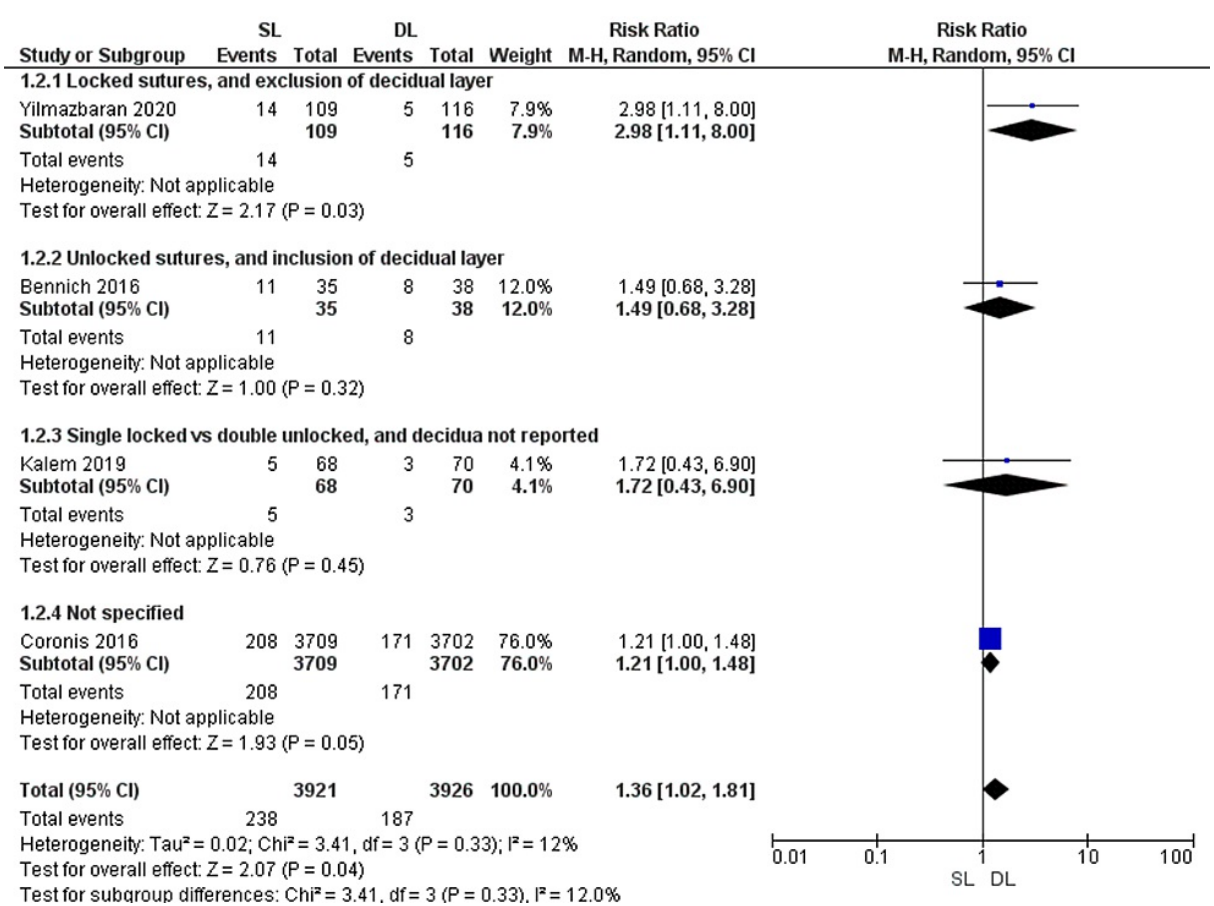

FIGURE 5: Forest plot comparing single- versus double-layer uterine closure in terms of dysmenorrhea.

$\mathrm{SL}$, single-layer uterine closure; $\mathrm{DL}$, double-layer uterine closure.

Uterine dehiscence or rupture (Figure 6)

The risk of uterine dehiscence or rupture was similar with SL and DL uterine closure $(\mathrm{RR}=1.88 ; 95 \%$ CI 0.63 , $5.62 ; \mathrm{P}=0.26)$. Pooled results were homogeneous $(\mathrm{P}=0.97 ; \mathrm{I} 2=0 \%)$.

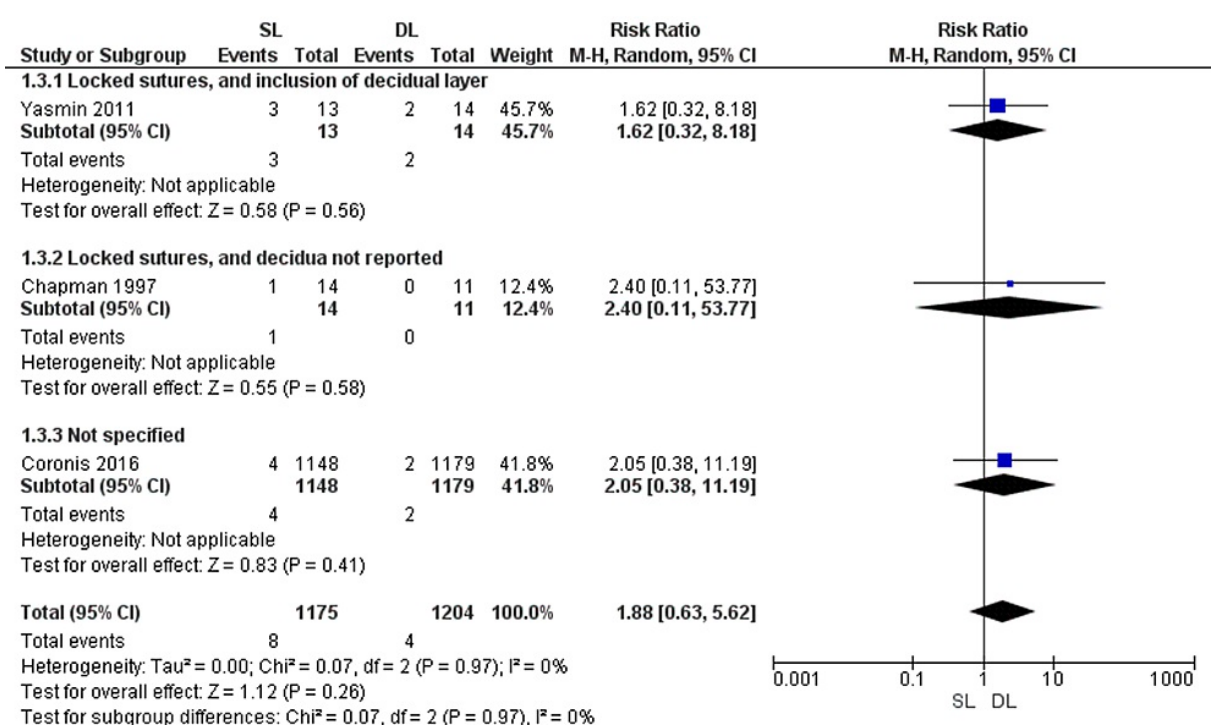

FIGURE 6: Forest plot comparing single- versus double-layer uterine closure in terms of uterine dehiscence or rupture.

$\mathrm{SL}$, single-layer uterine closure; $\mathrm{DL}$, double-layer uterine closure.

Healing ratio (Figure 7)

Healing ratio was comparable with SL and DL uterine closure (MD = -5.00; 95\% CI -12.40, 2.39; $\mathrm{P}=0.18$ ). 


\section{Cureus}

Pooled data were heterogeneous $(\mathrm{P}=0.005, \mathrm{I} 2=76 \%$ ). However, SL uterine closure showed lower healing ratio in the subgroup of locked sutures with inclusion of the decidual layer (MD $=-11.74 ; 95 \% \mathrm{CI}-21.43$, $2.05 ; \mathrm{P}=0.02)$, and pooled data were homogeneous $(\mathrm{P}=0.19 ; \mathrm{I} 2=43 \%)$.

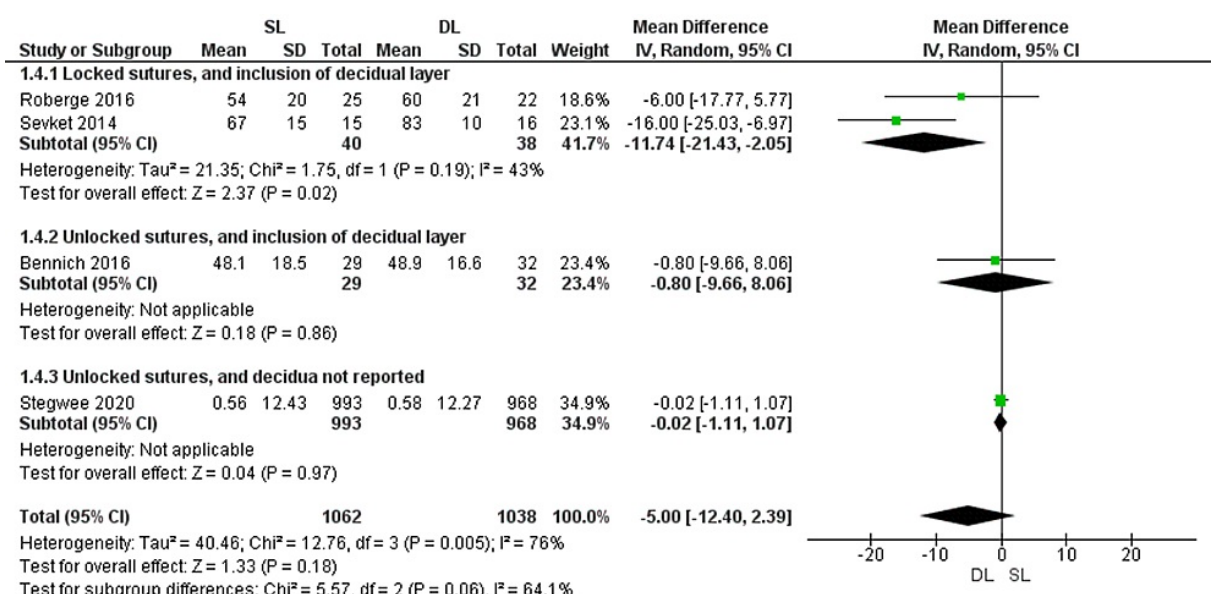

FIGURE 7: Forest plot comparing single- versus double-layer uterine closure in terms of healing ratio.

$\mathrm{SL}$, single-layer uterine closure; $\mathrm{DL}$, double-layer uterine closure.

Blood loss (Figure 8)

Pooled data showed that the amount of blood loss was comparable with SL and DL uterine closure $(\mathrm{MD}=$ 7.14; 95\% CI -16.21, 30.50; $\mathrm{P}=0.55)$. Pooled results were heterogeneous ( $\mathrm{P}=0.009 ; \mathrm{I} 2=61 \%)$. However, the subgroup analysis of patients who had locked sutures with inclusion of the decidua favored DL over SL uterine closure in the amount of blood loss ( $\mathrm{MD}=36.04 ; 95 \% \mathrm{CI} 13.05,59.03 ; \mathrm{P}=0.002)$, and the data were homogeneous ( $\mathrm{P}=0.49 ; \mathrm{I} 2=0 \%$ ). The subgroup who had unlocked sutures with inclusion of the decidual layer showed insignificant results $(\mathrm{MD}=12.12 ; 95 \% \mathrm{CI}-35.70,59.93 ; \mathrm{P}=0.62)$, and data were homogeneous $(\mathrm{P}=0.90 ; \mathrm{I} 2=0 \%)$. Also, the subgroup of unlocked sutures with no data about including the decidua showed no significant difference between SL and DL closure techniques (MD = -17.43; 95\% CI -36,07, 1.21; P = 0.07), and the results were homogeneous $(\mathrm{P}=0.27 ; \mathrm{I} 2=19 \%)$. 


\section{Cureus}

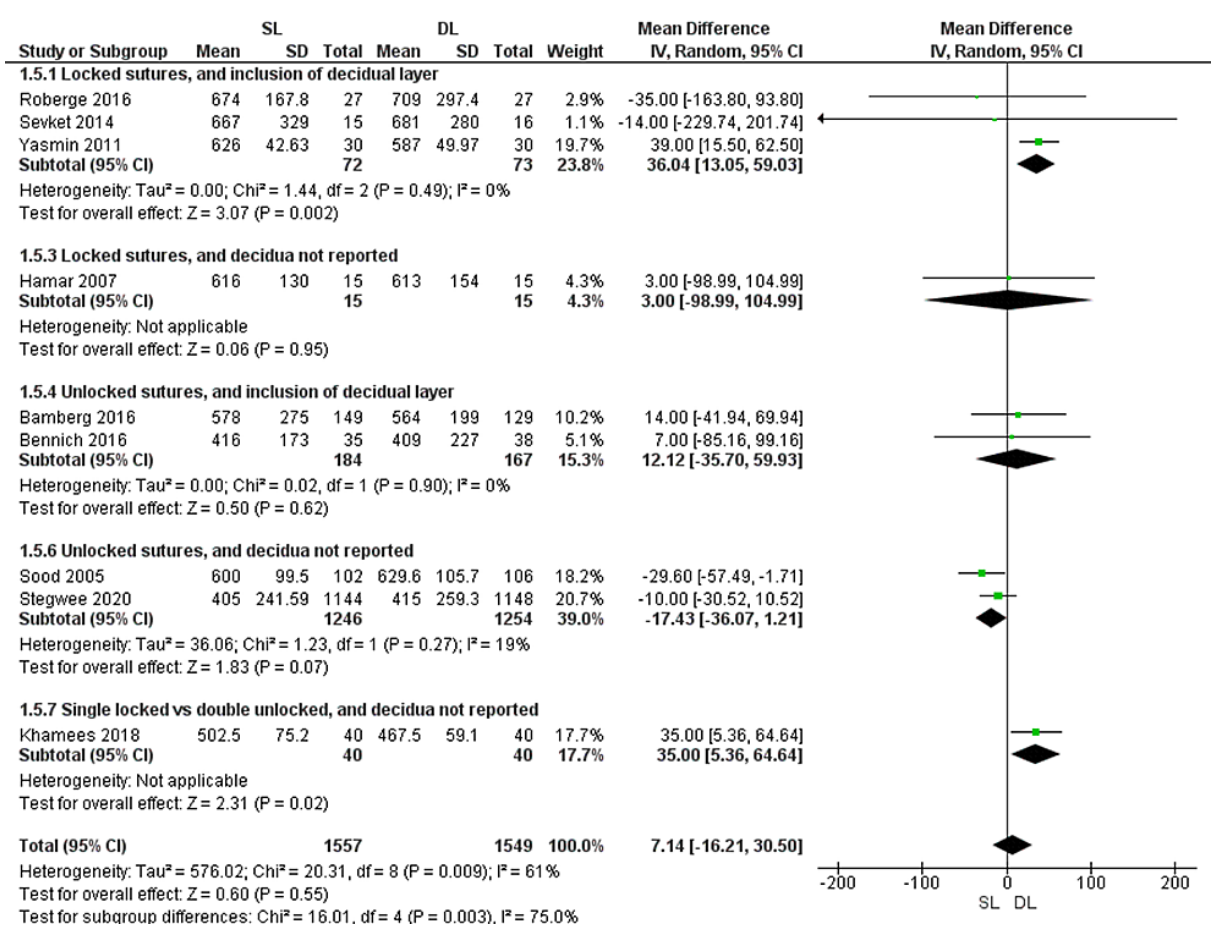

FIGURE 8: Forest plot comparing single- versus double-layer uterine closure in terms of blood loss.

$\mathrm{SL}$, single-layer uterine closure; $\mathrm{DL}$, double-layer uterine closure.

Operative time (Figure 9)

Pooled data showed that operative time is shorter with SL than with DL uterine closure $(\mathrm{MD}=-2.25 ; 95 \% \mathrm{CI}$ $-3.29,-1.21 ; \mathrm{P}<0.00001)$. Pooled results were heterogeneous $(\mathrm{P}<0.00001 ; \mathrm{I} 2=78 \%)$. Similar results were observed in the subgroup of locked sutures with no data about including the decidua (MD $=-3.78 ; 95 \%$ CI 5.83, - 1.74; $\mathrm{P}=0.0003$ ) (homogeneous data, $\mathrm{P}=0.87 ; \mathrm{I} 2=0 \%$ ), and in the subgroup of unlocked sutures with no data about including the decidua ( $\mathrm{MD}=-2.94 ; 95 \% \mathrm{CI}-4.99,-0.89 ; \mathrm{P}=0.005$ ) (heterogeneous data, $\mathrm{P}=$ $0.02 ; \mathrm{I} 2=94 \%)$. The difference between SL and DL closure was insignificant in the subgroup of unlocked sutures with inclusion of the decidua ( $\mathrm{MD}=-1.31 ; 95 \% \mathrm{CI}-2.89,0.26 ; \mathrm{P}=0.1$ ) (homogeneous data, $\mathrm{P}=0.67$; $\mathrm{I} 2=0 \%)$, and in the subgroup comparing SL closure using locked sutures versus DL closure using unlocked sutures $(\mathrm{MD}=-1.78 ; 95 \% \mathrm{CI}-7.46,3.91 ; \mathrm{P}=0.54)$ (heterogeneous data, $\mathrm{P}<0.0001 ; \mathrm{I} 2=94 \%$ ). 


\section{Cureus}

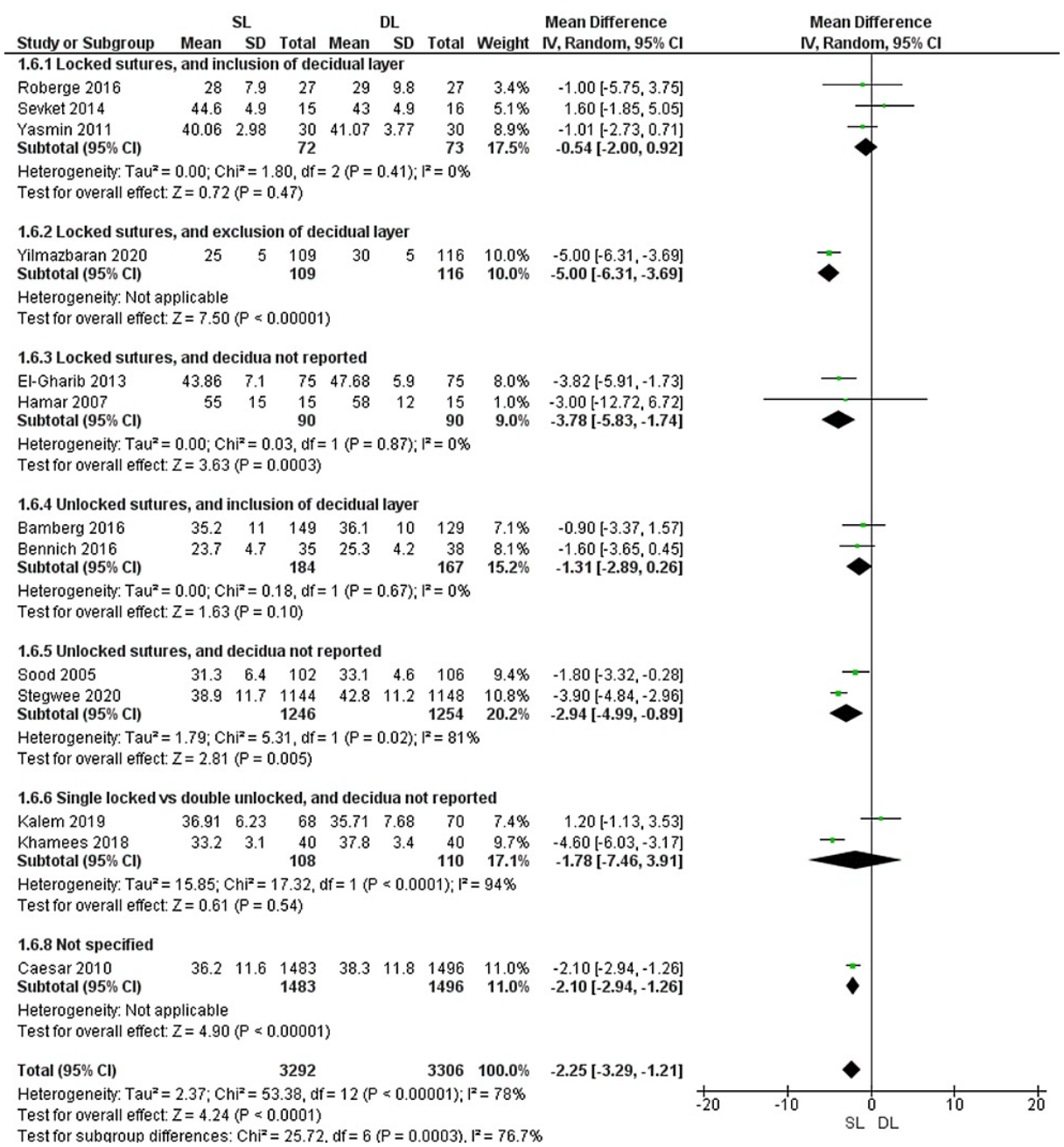

\section{FIGURE 9: Forest plot comparing single- versus double-layer uterine closure in terms of operative time.}

$\mathrm{SL}$, single-layer uterine closure; $\mathrm{DL}$, double-layer uterine closure.

Maternal infectious morbidity (Figure 10)

Pooled data showed no significant difference between SL and DL uterine closure in the risk of maternal infection morbidity $(\mathrm{RR}=0.94 ; 95 \% \mathrm{CI} 0.66,1.34 ; \mathrm{P}=0.72)$. Pooled results are heterogeneous $(\mathrm{P}=0.005, \mathrm{I} 2=$ $70 \%)$. Also, the difference was insignificant in the subgroups of unlocked sutures including the decidua $(\mathrm{RR}=$ 1.13 ; 95\% CI 0.43, 2.96; $\mathrm{P}=0.8$ ) (homogeneous data, $\mathrm{P}=0.84 ; \mathrm{I} 2=0 \%$ ) and the subgroup of locked sutures with no data about including the decidua $(\mathrm{RR}=1.27 ; 95 \% \mathrm{CI} 0.96,1.69 ; \mathrm{P}=0.1$ ) (homogeneous data, $\mathrm{P}=0.73$, I2 = 0\%). 


\section{Cureus}

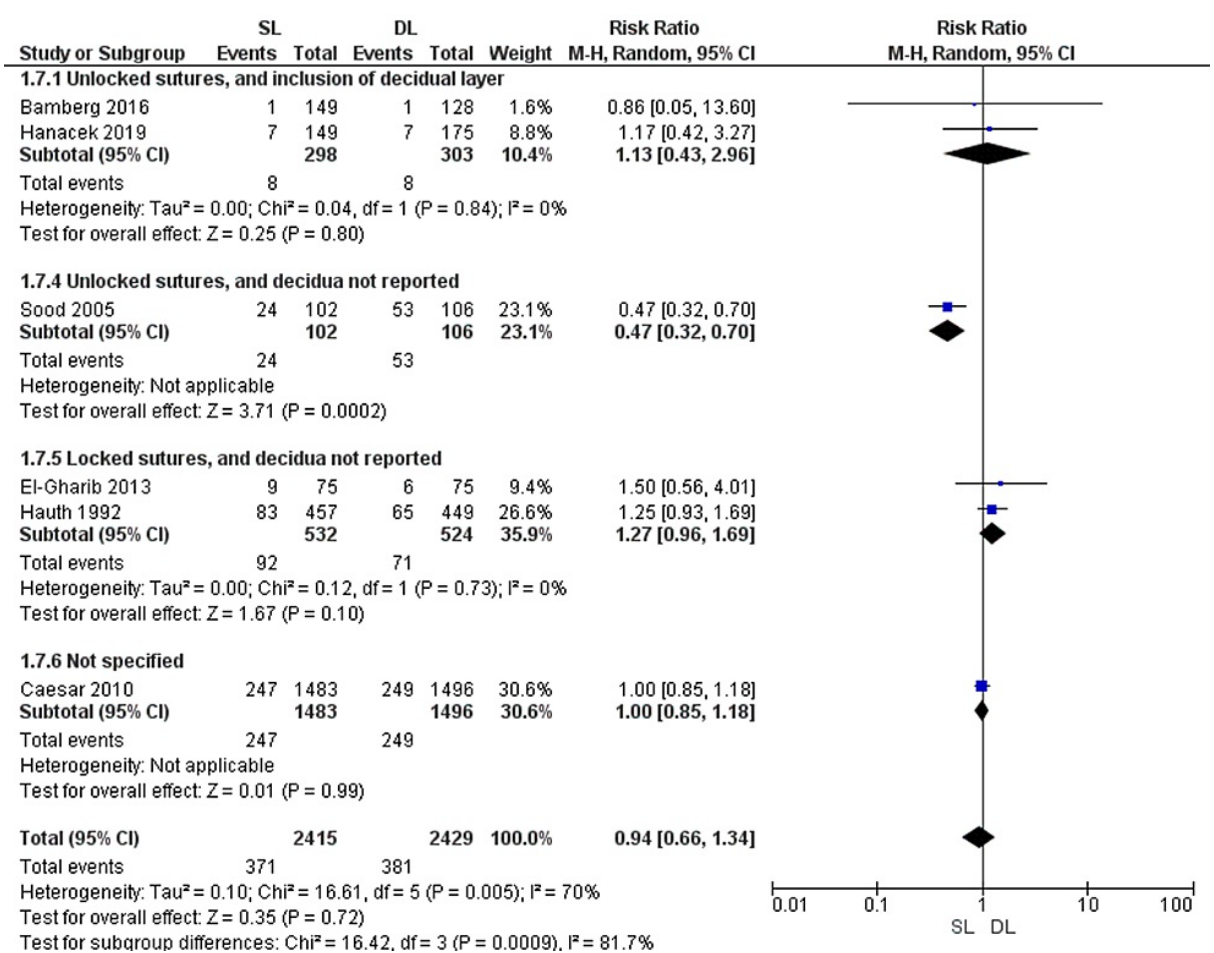

FIGURE 10: Forest plot comparing single- versus double-layer uterine closure in terms of maternal infectious morbidity.

$\mathrm{SL}$, single-layer uterine closure; $\mathrm{DL}$, double-layer uterine closure.

Hospital stay (Figure 11)

Pooled data showed no significant difference between SL and DL uterine closure in the period of hospital stay after procedure $(\mathrm{MD}=-0.12 ; 95 \% \mathrm{CI}-0.30,0.06 ; \mathrm{P}=0.18)$. Pooled results are heterogeneous $(\mathrm{P}=0.0003$ : I2 $=81 \%$ ). Also, the difference was insignificant in the subgroups of locked sutures with no data about including the decidua ( $\mathrm{MD}=-0.09 ; 95 \% \mathrm{CI}-0.34,0.16 ; \mathrm{P}=0.5$ ) (homogeneous data, $\mathrm{P}=0.54 ; \mathrm{I} 2=0 \%$ ) and the subgroup of unlocked sutures with no data about including the decidua (MD $=-0.25 ; 95 \% \mathrm{CI}-0.76,0.26 ; \mathrm{P}=$ 0.34 ) (heterogeneous data, $\mathrm{P}<0.00001, \mathrm{I} 2=95 \%$ ).

\begin{tabular}{|c|c|c|c|c|c|c|c|c|c|c|}
\hline \multirow[b]{2}{*}{ Study or Subgroup } & \multicolumn{3}{|c|}{ SL } & \multicolumn{3}{|c|}{ DL } & \multicolumn{2}{|r|}{ Mean Difference } & \multirow{2}{*}{\multicolumn{2}{|c|}{$\begin{array}{c}\text { Mean Difference } \\
\mathrm{N}, \text { Random, } 95 \% \mathrm{Cl}\end{array}$}} \\
\hline & Mean & SD & Total & Mean & SD & Total & Weight & IV, Random, $95 \% \mathrm{Cl}$ & & \\
\hline \multicolumn{11}{|c|}{ 1.8.1 Locked sutures, and decidua not reported } \\
\hline Chapman 1997 & 3.1 & 2 & 70 & 3 & 2 & 75 & $6.0 \%$ & $0.10[-0.55,0.75]$ & & \\
\hline El-Gharib 2013 & 1.48 & 0.8 & 75 & 1.6 & 0.9 & 75 & $17.8 \%$ & $-0.12[-0.39,0.15]$ & & \\
\hline Subtotal $(95 \% \mathrm{Cl})$ & & & 145 & & & 150 & $23.8 \%$ & $-0.09[-0.34,0.16]$ & & \\
\hline \multicolumn{11}{|c|}{$\begin{array}{l}\text { Heterogeneity. Tau }=0.00 ; C h i^{2}=0.37, d f=1(P=0.54) ; I^{2}=0 \% \\
\text { Test for overall effect: } Z=0.68(P=0.50)\end{array}$} \\
\hline \multicolumn{11}{|c|}{ 1.8.2 Unlocked sutures, and decidua not reported } \\
\hline Sood 2005 & 6.67 & 0.81 & 102 & 7.19 & 0.85 & 106 & $20.5 \%$ & $-0.52[-0.75,-0.29]$ & $\rightarrow-$ & \\
\hline $\begin{array}{l}\text { Stegwee } 2020 \\
\text { Subtotal }(95 \% \mathrm{Cl})\end{array}$ & 3 & 0.33 & 1144 & 3 & 0.17 & 1148 & $30.3 \%$ & $0.00[-0.02,0.02]$ & & \\
\hline \multirow{2}{*}{\multicolumn{11}{|c|}{$\begin{array}{l}\text { Heterogeneity: } \text { Tau }^{2}=0.13 ; \mathrm{Chi}^{2}=20.23, \mathrm{df}=1(P<0.00001) ; \mathrm{I}^{2}=95 \% \\
\text { Test for overall effect: } Z=0.95(P=0.34)\end{array}$}} \\
\hline & & & & & & & & & & \\
\hline \multicolumn{11}{|l|}{ 1.8.3 Not specified } \\
\hline $\begin{array}{l}\text { Caesar } 2010 \\
\text { Subtotal } 95 \% \text { ClI }\end{array}$ & 4.7 & 2 & 1483 & 4.7 & 2 & 1496 & $25.4 \%$ & $0.00[-0.14,0.14]$ & & \\
\hline \multirow{2}{*}{\multicolumn{11}{|c|}{$\begin{array}{l}\text { Heterogeneity: Not applicable } \\
\text { Test for overall effect: } Z=0.00(P=1.00)\end{array}$}} \\
\hline & & & & & & & & & & \\
\hline Total $(95 \% \mathrm{Cl})$ & & & 2874 & & & 2900 & $100.0 \%$ & $-0.12[-0.30,0.06]$ & & \\
\hline $\begin{array}{l}\text { Heterogeneity: Tauª } \\
\text { Test for overall effect }\end{array}$ & $\begin{array}{l}0.03 ; C h \\
Z=1.34\end{array}$ & $\begin{array}{l}i^{2}=2 \\
(P=0\end{array}$ & $\begin{array}{ll}1.02, \mathrm{df} \\
18)\end{array}$ & & & & $81 \%$ & & $\mathrm{SL}^{0} \mathrm{DL}$ & $\frac{1}{2}$ \\
\hline
\end{tabular}

FIGURE 11: Forest plot comparing single- versus double-layer uterine closure in terms of hospital stay.

$\mathrm{SL}$, single-layer uterine closure; $\mathrm{DL}$, double-layer uterine closure. 
In this systematic review and meta-analysis, we included RCTs only to provide high-quality class-one evidence and followed the widely accepted PRISMA guidelines during the conduction of this study. We included all published RCTs with no publication date restriction. In addition, we performed subgroup analyses according to variations in the surgical techniques (locked or unlocked sutures, and inclusion or exclusion of the decidua) to solve the heterogeneity between studies. Limitations in this study include the heterogeneity detected in many outcomes and could not be solved in some cases. In addition, some longterm outcomes were reported by a small number of studies, which limits the generalizability of the results. Future studies with large sample size and longer follow-up would provide more conclusive results.

\section{Conclusions}

DL uterine closure technique was associated with more RMT compared with SL closure technique. Also, patients who had DL uterine closure showed lower incidence of dysmenorrhea. On the other hand, SL closure was associated with significantly shorter operation time. Both techniques showed comparable healing ratio, readmission rate, and hospital stay. Also, the amount of blood loss, the risk of maternal infection, and the risk of uterine dehiscence or rupture during a subsequent delivery were similar with both techniques.

\section{Additional Information \\ Disclosures}

Conflicts of interest: In compliance with the ICMJE uniform disclosure form, all authors declare the following: Payment/services info: All authors have declared that no financial support was received from any organization for the submitted work. Financial relationships: All authors have declared that they have no financial relationships at present or within the previous three years with any organizations that might have an interest in the submitted work. Other relationships: All authors have declared that there are no other relationships or activities that could appear to have influenced the submitted work.

\section{Acknowledgements}

Kaif Qayum and Irfan Kar contributed equally to this study. Therefore, joint first-authorship is proposed.

\section{References}

1. Boerma T, Ronsmans C, Melesse DY, et al.: Global epidemiology of use of and disparities in caesarean sections. Lancet. 2018, 392:1341-8. 10.1016/s0140-6736(18)31928-7

2. Sandall J, Tribe RM, Avery L, et al.: Short-term and long-term effects of caesarean section on the health of women and children. Lancet. 2018, 392:1349-57. 10.1016/s0140-6736(18)31930-5

3. Abalos E, Addo V, Brocklehurst P, et al.: Caesarean section surgical techniques: 3 year follow-up of the CORONIS fractional, factorial, unmasked, randomised controlled trial. Lancet. 2016, 388:62-72. 10.1016/S0140-6736(16)00204-X

4. Bij de Vaate AJ, van der Voet LF, Naji O, et al.: Prevalence, potential risk factors for development and symptoms related to the presence of uterine niches following Cesarean section: systematic review. Ultrasound Obstet Gynecol. 2014, 43:372-82. 10.1002/uog.13199

5. Kok N, Wiersma IC, Opmeer BC, de Graaf IM, Mol BW, Pajkrt E: Sonographic measurement of lower uterine segment thickness to predict uterine rupture during a trial of labor in women with previous Cesarean section: a meta-analysis. Ultrasound Obstet Gynecol. 2013, 42:132-9. 10.1002/uog.12479

6. van der Voet LF, Bij de Vaate AM, Veersema S, Brölmann HA, Huirne JA: Long-term complications of caesarean section. The niche in the scar: a prospective cohort study on niche prevalence and its relation to abnormal uterine bleeding. BJOG. 2014, 121:236-44. 10.1111/1471-0528.12542

7. Roberge S, Demers S, Berghella V, Chaillet N, Moore L, Bujold E: Impact of single- vs double-layer closure on adverse outcomes and uterine scar defect: a systematic review and metaanalysis. Am J Obstet Gynecol. 2014, 211:453-60. 10.1016/j.ajog.2014.06.014

8. Stegwee SI, van der Voet LF, Ben AJ, et al.: Effect of single- versus double-layer uterine closure during caesarean section on postmenstrual spotting (2Close): multicentre, double-blind, randomised controlled superiority trial. BJOG. 2021, 128:866-78. 10.1111/1471-0528.16472

9. Stegwee SI, Jordans I, van der Voet LF, et al.: Uterine caesarean closure techniques affect ultrasound findings and maternal outcomes: a systematic review and meta-analysis. BJOG. 2018, 125:1097-108. 10.1111/1471-0528.15048

10. Higgins JP, Thomas J, Chandler J, Cumpston M, Li T, Page MJ, Welch VA: Cochrane Handbook for Systematic Reviews of Interventions. 2nd Edition. John Wiley \& Sons, Chichester, UK; 2019.

11. Liberati A, Altman DG, Tetzlaff J, et al.: The PRISMA statement for reporting systematic reviews and metaanalyses of studies that evaluate health care interventions: explanation and elaboration. PLoS Med. 2009, 6:e1000100. 10.1371/journal.pmed.1000100

12. Higgins JP, Altman DG, Gøtzsche PC, et al.: The Cochrane Collaboration's tool for assessing risk of bias in randomised trials. BMJ. 2011, 343:d5928. 10.1136/bmj.d5928

13. The CAESAR study collaborative group: Caesarean section surgical techniques: a randomised factorial trial (CAESAR). BJOG. 2010, 117:1366-76. 10.1111/j.1471-0528.2010.02686.x

14. Bamberg C, Dudenhausen JW, Bujak V, et al.: A prospective randomized clinical trial of single vs. double layer closure of hysterotomy at the time of cesarean delivery: the effect on uterine scar thickness. Ultraschall Med. 2018, 39:343-51. 10.1055/s-0042-112223

15. Bennich G, Rudnicki M, Wilken-Jensen C, Lousen T, Lassen PD, Wøjdemann K: Impact of adding a second 
layer to a single unlocked closure of a Cesarean uterine incision: randomized controlled trial. Ultrasound Obstet Gynecol. 2016, 47:417-22. 10.1002/uog.15792

16. Chapman SJ, Owen J, Hauth JC: One- versus two-layer closure of a low transverse cesarean: the next pregnancy. Obstet Gynecol. 1997, 89:16-8. 10.1016/s0029-7844(97)84257-3

17. Khamees RE, Khedr AH, Shaaban M, Bahi-Eldin M: Effect of single versus double layer suturing on healing of uterine scar after cesarean delivery. Suez Canal Univ Med J. 2018, 21:140-5. 10.21608/scumj.2018.43594

18. ELGharib MN, Awara AM: Ultrasound evaluation of the uterine scar thickness after single versus double layer closure of transverse lower segment cesarean section. J Basic Clin Reprod Sci. 2013, 2:42-5. 10.4103/2278-960X.112591

19. Hamar BD, Saber SB, Cackovic M, et al.: Ultrasound evaluation of the uterine scar after cesarean delivery: a randomized controlled trial of one- and two-layer closure. Obstet Gynecol. 2007, 110:808-13. 10.1097/01.AOG.0000284628.29796.80

20. Hanacek J, Vojtech J, Urbankova I, Krcmar M, Křepelka P, Feyereisl J, Krofta L: Ultrasound cesarean scar assessment one year postpartum in relation to one- or two-layer uterine suture closure. Acta Obstet Gynecol Scand. 2020, 99:69-78. 10.1111/aogs.13714

21. Hauth JC, Owen J, Davis RO: Transverse uterine incision closure: one versus two layers . Am J Obstet Gynecol. 1992, 167:1108-11. 10.1016/s0002-9378(12)80048-2

22. Kalem Z, Kaya AE, Bakırarar B, Basbug A, Kalem MN: An optimal uterine closure technique for better scar healing and avoiding isthmocele in cesarean section: a randomized controlled study. J Invest Surg. 2021, 34:148-56. 10.1080/08941939.2019.1610530

23. Kumar SA: Single versus double layer closure of low transverse uterine incision at cesarean section . J Obstet Gynecol India. 2005, 55:231-6.

24. Roberge S, Demers S, Girard M, et al.: Impact of uterine closure on residual myometrial thickness after cesarean: a randomized controlled trial. Am J Obstet Gynecol. 2016, 214:507.e1-6. 10.1016/j.ajog.2015.10.916

25. Sevket O, Ates S, Molla T, Ozkal F, Uysal O, Dansuk R: Hydrosonographic assessment of the effects of 2 different suturing techniques on healing of the uterine scar after cesarean delivery. Int J Gynaecol Obstet. 2014, 125:219-22. 10.1016/j.ijgo.2013.11.013

26. Shrestha P, Shrestha S, Gyawali M: Ultrasound evaluation of uterine scar in primary caesarean section: a study of single versus double layer uterine closure. Am J Public Health Res. 2015, 3:178-81. 10.12691/ajphr3-5A-37

27. Yasmin S, Sadaf J, Fatima N: Impact of methods for uterine incision closure on repeat caesarean section scar of lower uterine segment. J Coll Physicians Surg Pak. 2011, 21:522-6.

28. Yılmaz Baran Ş, Kalaycı H, Doğan Durdağ G, Yetkinel S, Alemdaroğlu S, Çok T, Bulgan Kılıçdağ E: Single- or double-layer uterine closure techniques following cesarean: A randomized trial. Acta Obstet Gynecol Scand. 2021, 100:531-7. 10.1111/aogs.14018

29. Hesselman S, Högberg U, Ekholm-Selling K, Råssjö EB, Jonsson M: The risk of uterine rupture is not increased with single- compared with double-layer closure: a Swedish cohort study. BJOG. 2015, 122:153541. 10.1111/1471-0528.13015

30. Menacker F, Declercq E, Macdorman MF: Cesarean delivery: background, trends, and epidemiology. Semin Perinatol. 2006, 30:235-41. 10.1053/j.semperi.2006.07.002 